\title{
Lattice dynamics of rhenium trioxide from the quasiharmonic approximation
}

\author{
U. D. Wdowik and K. Parlinski \\ Institute of Technology, Pedagogical University, ul. Podchorazych 2, 30-084 Cracow, Poland \\ Tapan Chatterji \\ Jülich Center for Neutron Science, Forschungszentrum Jülich Outstation at Institute Laue-Langevin, BP 156, \\ 38042 Grenoble Cedex 9, France \\ S. Rols and H. Schober \\ Institute Laue-Langevin, BP 156, 38042 Grenoble Cedex 9, France
}

(Received 26 May 2010; revised manuscript received 2 August 2010; published 10 September 2010)

\begin{abstract}
The quasiharmonic theory is applied to study the lattice dynamics and thermal properties of rhenium trioxide, a material exhibiting the negative thermal-expansion phenomenon. Phonons are calculated at several external pressures. The pressure dependence of the $M, R$, and zone-center phonon modes is analyzed. Relying on the Grüneisen formalism an influence of temperature on the $M$ phonon mode is investigated. The calculated free energy of the system provides predictions for the temperature dependence of such quantities as equilibrium volume and crystal thermal expansivity. The mean-squared vibrations of rhenium-trioxide atoms are investigated versus temperature and pressure. Results of the calculations indicate a softening of the $M$ and $R$ phonons with pressure and a large negative Grüneisen constants for these modes. A decrease in the crystal volume is observed up to $170 \mathrm{~K}$. The thermal-expansion coefficient shows a minimum in the vicinity of $100 \mathrm{~K}$ which corresponds to a minimum of the anisotropy in the oxygen thermal vibrations perpendicular to the Re-O bond and along the bond. Anisotropy in the oxygen Debye-Waller factors follows from the anisotropy of the force constants at the oxygen site. Inelastic neutron-scattering measurements performed at room temperature are reported together with the generalized phonon densities of states. A comparison between present calculations and the experimental data as well as other theoretical studies is provided.
\end{abstract}

DOI: 10.1103/PhysRevB.82.104301

PACS number(s): 63.20.dk, 65.40.-b, 61.05.F-

\section{INTRODUCTION}

Materials exhibiting negative thermal expansion (NTE) have been attracting significant attention for the last few years particularly due to their potential industrial applications. ${ }^{1}$ On the other hand, these materials also show many other unusual physicochemical properties which make them interesting from a fundamental point of view.

The NTE phenomenon can originate from different mechanisms involving magnetostriction in ferroelectric materials, ${ }^{2}$ valence transition in intermetallic compounds ${ }^{3}$ and fulleride materials. ${ }^{4}$ Some class of lattice vibrations, e.g., low-frequency rigid unit modes in oxide-based framework materials $^{5,6}$ or cyanide group-based framework materials ${ }^{7-9}$ can be responsible for the NTE behavior as well.

The most extensively studied NTE materials with the open framework structure (oxides or $\mathrm{CN}$-based compounds) are insulators or semiconductors. Rhenium trioxide, $\mathrm{ReO}_{3}$, is unique among such compounds since it is a metal with remarkably low electrical resistivity ${ }^{10,11}$ being comparable to that of cooper or silver. The metallic conductivity of $\mathrm{ReO}_{3}$ is assigned to delocalization of $5 d$ electrons of Re atom. Despite the metallic behavior, the properties of the $\mathrm{ReO}_{3}$ lattice are not typical for a metallic phase. The $\mathrm{Re}-\mathrm{O}$ bond formed by the hybridized $\operatorname{Re} 5 d$ and $\mathrm{O} 2 p$ states is highly covalent $^{12-14}$ and therefore this oxide is sometimes called a covalent metal.

Rhenium trioxide crystallizes in a cubic space group $\left(P m 3 m, O_{h}^{1}\right)$ with a lattice constant of $3.74 \AA$. The structure consists of the vortex-sharing $\mathrm{ReO}_{6}$ octahedra and linear $\mathrm{Re}$ -
O-Re chains with Re atoms located at the center of each octahedron, as shown in Fig. $1 . \mathrm{ReO}_{3}$ belongs to the family of perovskite-type compounds. This family comprises ternary and binary transition-metal oxides with the general formula of $\mathrm{ABO}_{3}$ and $\mathrm{BO}_{3}$, respectively. In the ternary systems, the 12-fold coordinated sites of the oxygen sublattice are occupied by large cations of A type while the smaller cations of B type are coordinated octahedrally by oxygen atoms forming corner-sharing network of $\mathrm{BO}_{6}$ octahedra. In binary compounds such as $\mathrm{ReO}_{3}$ the A site is vacant. This allows for a rigid rotation of the $\mathrm{ReO}_{6}$ units around [100]-type axes and

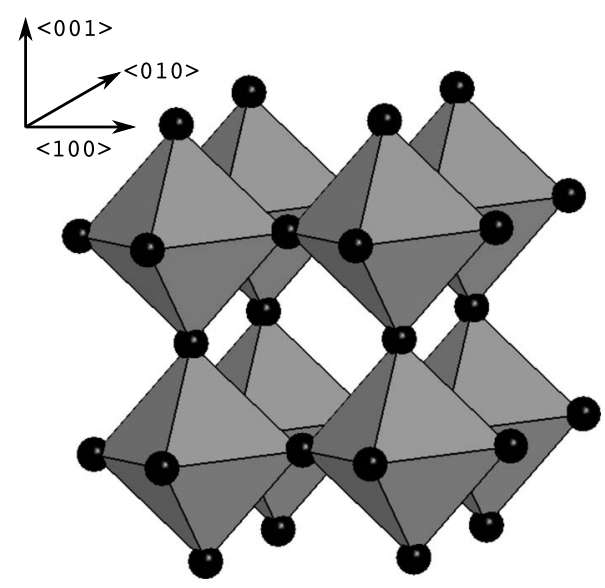

FIG. 1. Schematic view of the corner-sharing network of $\mathrm{ReO}_{3}$ octahedra. Rhenium atom occupies center of each octahedron. 


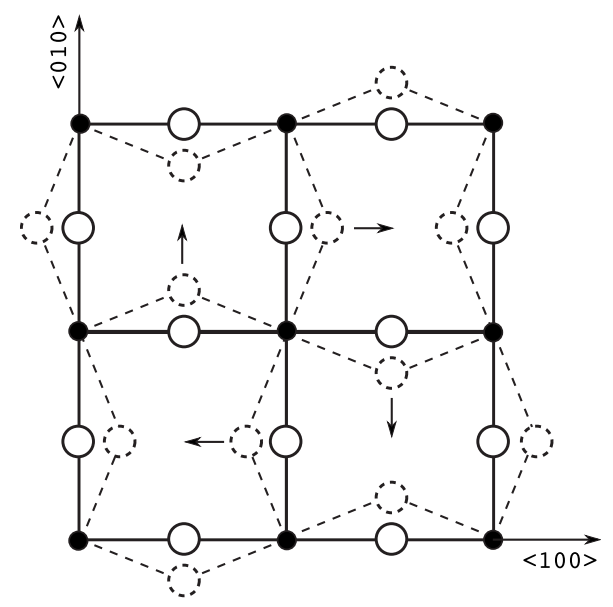

FIG. 2. Schematic view of the $M$-type phonon mode involving rigid rotations of $\mathrm{ReO}_{6}$ octahedra along [100]-type axes. Rhenium and oxygen atoms are shown as solid and open circles, respectively.

can result in the anisotropy of the thermal motion of anions. ${ }^{15}$ The rigid unit modes can also lead to NTE phenomenon. ${ }^{15,16}$ The rotations of $\mathrm{ReO}_{3}$ octahedra are expected to be responsible for the experimentally observed structural phase transition occurring at elevated pressures. ${ }^{17-28}$ The cubic $P m \overline{3} m$ structure of $\mathrm{ReO}_{3}$ transforms to cubic Im 3 structure at about $5.2 \mathrm{kbar}$ at room temperature. ${ }^{20-22}$ The transition pressure decreases upon cooling and it amounts to $3.5 \mathrm{kbar}$ at $150 \mathrm{~K}$ (Ref. 28) and 2.5 kbar at liquid-He temperature (Ref. 18). Some experiments $^{21,22}$ suggest an existence of the intermediate tetragonal phase $(P 4 / \mathrm{mbm})$ or a sequence of phases $(P 4 / \mathrm{mbm} \rightarrow I 4 / \mathrm{mmm})$ occurring between 5 and $5.3 \mathrm{kbar}$. Such phase/phases having a very narrow stability range were not confirmed by the recent neutron-diffraction measurements. ${ }^{27}$ The latter experiments suggest a direct transition from $P m \overline{3} m$ to $\operatorname{Im} \overline{3}$ phase which occurs below $300 \mathrm{~K}$. The pressure-induced phase transition was explained as to be driven by the softening of the $M$-type phonon mode with $\mathrm{ReO}_{3}$ compression. This mode is schematically shown in Fig. 2. Oxygen atoms which lie directly between neighboring Re atoms are shifted from their equilibrium positions and vibrate perpendicular to the $\mathrm{Re}-\mathrm{O}-\mathrm{Re}$ bonds. Such a movement can be considered as the coordinated rotations of the neighboring octahedra around [100]-type axes. Adjacent octahedra rotate as rigid units in opposite direction to each other. Therefore, a nanoscale structure of $\mathrm{ReO}_{3}$ seems to be locally distorted and it appears cubic only for methods operating on spatial averaged structures.

The rigid unit modes, responsible for the pressure-induced structural phase transition in the $\mathrm{ReO}_{3}$ compound, may also account for the NTE phenomenon observed in this material at low temperatures. An inconsistency in the experimental data concerning the thermal expansivity of $\mathrm{ReO}_{3}$ has to be noted. A laser interferometry experiments ${ }^{29}$ point out NTE behavior between 100 and $340 \mathrm{~K}$, the X-ray diffraction studies and extended x-ray absorption fine structure spectroscopy $^{30}$ report NTE below $100 \mathrm{~K}$ while in the neutron-diffraction measurements ${ }^{15}$ the NTE is observed up to $200 \mathrm{~K}$. The most recent neutron-diffraction studies ${ }^{31}$ indi- cate that transition from negative to positive thermal expansivity significantly depends on the sample quality. The highest quality samples of $\mathrm{ReO}_{3}$ reveal less static disorder, i.e., the NTE phenomenon can exist even at room temperature.

The present work extends a description of $\mathrm{ReO}_{3}$ compound to finite temperatures using the approach of the quasiharmonic approximation and supplements previous lattice dynamical calculations. ${ }^{15}$ Some selected thermal properties of this material are investigated and compared to the available experimental data and other theoretical studies which were based on the phenomenological models. Special attention is paid to a behavior of the $M$ phonon mode versus pressure and the crystal thermal expansivity. Additionally, a detailed analysis of the thermal motion of particular atoms constituting the $\mathrm{ReO}_{3}$ lattice is presented since it is expected to provide an insight on the origin of the negative thermal expansivity in this material.

\section{EXPERIMENTAL AND CALCULATION}

\section{A. Inelastic neutron-scattering experiments}

Inelastic neutron-scattering measurements were performed using the IN4C spectrometer at the Institut Laue Langevin in Grenoble. IN4C is a time of flight instrument installed on a thermal beam providing a high neutron flux in the wavelength range from 0.8 to $3.6 \AA$. The neutron released from the $\mathrm{D}_{2} \mathrm{O}$ moderator are monochromatized by a vertically and horizontally curved graphite monochromator. The beam is thereafter sharply pulsed by a Fermi chopper placed close to the sample position. The scattered neutrons have their energy $E_{f}$ and wave vector $\overrightarrow{k_{f}}$ analyzed by the time of flight technique: after scattering by the sample, they "fly" inside an evacuated "flight chamber" having a $2 \mathrm{~m}$ diameter and covered by $330 \mathrm{He}^{3}$ detectors allowing detection for scattering angles from $2.5^{\circ}$ to $122^{\circ}$. The time the neutrons spend flying from the sample to the detector is then transformed into a velocity and therefore into energy transfer $\hbar \omega=E_{i}-E_{f}$ between the neutron and the sample. In our experiment, the incident neutron energy was fixed at $E_{i}$ $=16.9 \mathrm{meV}(2.2 \AA)$ and the instrument was setup to have the best performance in the neutron energy gain side of the spectrum (anti-Stokes), which requires a speed for the Fermi rotation of $20000 \mathrm{rpm}$. In this configuration, the resolution of the spectrometer is $\sim 0.6 \mathrm{meV}$ at elastic scattering, $\sim 4 \mathrm{meV}$ at $50 \mathrm{meV}$, and reaching $\sim 10 \mathrm{meV}$ at $100 \mathrm{meV}$. The time of flight data measured for the $\mathrm{ReO}_{3}$ sample are reduced by normalizing to the monitor counts by correcting from the background and container (cylindrical aluminum can) contributions and by correcting for detector efficiency.

In the case of a crystalline powder of incoherent scatterer, one obtains the incoherent scattering cross section which-in the assumption of small amplitude for the atomic vibrations - can be expressed as ${ }^{32}$

$$
\left(\frac{\partial^{2} \sigma}{\partial \Omega \partial \omega}\right)_{i n c}=\frac{k_{f}}{k_{i}} \frac{n(\omega) Q^{2}}{8 \pi \omega} \sum_{\mu} \frac{\sigma_{\mu}^{i}}{M_{\mu}} e^{-2 W_{\mu}(Q)} g_{\mu}(\omega)
$$

with 


$$
g_{\mu}(\omega)=\sum_{\vec{k}, j}|\vec{e}(\vec{k}, j \mid \mu)|^{2} \delta[\omega-\omega(\vec{k}, j)]
$$

being defined as the partial density of states of the $\mu$ th atom in the cell. In Eq. (1), $n(\omega)$ is the Bose population factor of states with energy $\hbar \omega$ and $Q$ is the value of the scattering vector $\vec{Q}=\overrightarrow{k_{i}}-\overrightarrow{k_{f}}$. The quantity $\vec{e}(\vec{k}, j \mid \mu)$ is the polarization vector of atom $\mu$ (of mass $M_{\mu}$ and incoherent scattering cross section $\left.\sigma_{\mu}^{i}\right)$ for the phonon $(\vec{k}, j)$. The term $2 W_{\mu}(Q)$ refers to the Debye-Waller factor associated to the atomic species $\mu$. By further integrating the scattering cross section given in Eq. (1) over $Q$, i.e., by summing the counts in each detector as a function of energy transfer weighted by $\sin (\theta)$, one obtains

$$
\begin{aligned}
\int_{\theta_{\min }}^{\theta_{\max }}\left(\frac{\partial^{2} \sigma}{\partial \Omega \partial \omega}\right)_{\text {inc }} \sin (\theta) d \theta= & \int_{Q_{\min }^{Q_{\max }}}\left(\frac{\partial^{2} \sigma}{\partial \Omega \partial \omega}\right)_{i n c} Q d Q \\
& =\frac{e^{-2 W}}{32 \pi k_{i}^{2}}\left(Q_{\max }^{4}-Q_{\min }^{1}\right) \\
& \times \bar{\alpha} \frac{n(\omega)}{\omega} G(\omega)
\end{aligned}
$$

with the generalized density of states (GDOS) given as

$$
G(\omega)=\sum_{\mu} \frac{\alpha_{\mu}}{\bar{\alpha}} g_{\mu}(\omega), \quad \alpha_{\mu}=\frac{\sigma_{\mu}^{i}}{M_{\mu}}, \quad \bar{\alpha}=\sum_{\mu} \alpha_{\mu} .
$$

In the previous integration, it is assumed that the DebyeWaller factor $2 W$ is independent of the atomic species and one neglects its $Q$ dependence. It is as well assumed that the multiphonon scattering can be neglected. In our experiment, due to the large values of $Q$ involved in the scattering, the multiphonon contribution can give an important background in the measured spectrum, especially at high energy transfer. The experimental spectrum can be corrected from both multiphonon and Debye-Waller contributions by using an iterative algorithm which is contained in the computer program MUPHOCOR, developed at Karlsruhe. ${ }^{33}$ The final quantity measured is expressed as

$$
\left(\frac{\partial \sigma}{\partial \omega}\right)_{i n c}^{1 p}=\frac{1}{32 \pi k_{i}^{2}}\left(Q_{\max }^{4}-Q_{\min }^{4}\right) \bar{\alpha} \frac{n(\omega)}{\omega} G(\omega)
$$

from which $G(\omega)$ can be extracted.

The same formalism can be applied to coherent scatterers $^{34}$ (such as Re and $\mathrm{O}$ ) providing that the scattering implies a large panel of scattering vectors $\vec{Q}=\overrightarrow{k_{i}}-\overrightarrow{k_{f}}$ which is the case for IN4C owing to the large coverage in scattering angle and to the relatively short incident wavelength used. The multiphonon corrections to $G(\omega)$ were made up to tenthorder multiphonon processes.

\section{B. Density-functional theory calculations}

$A b$ initio calculations of $\mathrm{ReO}_{3}$ crystal have been performed within the density-functional theory and by using the pseudopotential method with the local-density approximation (LDA) implemented in the VASP code. ${ }^{35}$ Atoms were represented by the projector-augmented wave (PAW) pseudopo- tentials provided by VASP. These pseudopotentials represent $\left(6 s^{2} 5 d^{5}\right)$ and $\left(s^{2} p^{4}\right)$ electron configurations of Re and $\mathrm{O}$ atoms, respectively. A plane-wave expansion up to $520 \mathrm{eV}$ was used. All calculations were done with $2 \times 2 \times 2$ supercell containing 32 atoms. The Brillouin zone was sampled using the $4 \times 4 \times 4 k$-point mesh generated by the Monkhorst-Pack scheme. A combination of conjugate gradient energy minimization and a quasi-Newton force minimization was used to optimize geometry and the atomic positions of the supercell. The atomic positions were relaxed until the forces were smaller than $10^{-6} \mathrm{eV} / \AA$, while the total energy was converged down to $10^{-7} \mathrm{eV}$.

Dynamical properties of $\mathrm{ReO}_{3}$ crystal were calculated using the direct method ${ }^{36}$ based on the forces calculated via Hellmann-Feynman (HF) theorem. The nonvanishing HF forces acting on the atoms in a given supercell were generated by displacing crystallographically nonequivalent $R e$ and $\mathrm{O}$ atoms from their equilibrium positions. Due to the symmetry of $\mathrm{ReO}_{3}$ crystal one and two displacements are required for $\mathrm{Re}$ and $\mathrm{O}$ atoms, respectively. To minimize systematic error, both positive and negative displacements were applied and therefore 2 and 4 displacements were calculated for $\mathrm{Re}$ and $\mathrm{O}$ atoms, respectively. The displacement amplitude of $0.03 \AA$ a was used. Phonons were calculated for $\mathrm{ReO}_{3}$ crystal exposed to external pressures ranging from -30 to 30 kbar.

To study the thermal properties of the crystal the quasiharmonic approximation ${ }^{37}$ was applied. In this approach a change in the crystal volume as a function of temperature is mapped to the change in the crystal volume at $T=0 \mathrm{~K}$ (typically as a function of pressure). Thermodynamical functions are calculated using standard formula for harmonic crystal. Anharmonic effects are to some extent taken into account by the volume (pressure) dependence of the phonon frequencies. Phonon frequencies at the constant volume are assumed to be independent of temperature and the relative change in the $(\vec{k}, j)$ mode frequency $\omega(\vec{k}, j)$ with volume $V$ is usually described by the mode-specific Grüneisen parameter which is a dimensionless quantity defined as ${ }^{37}$

$$
\gamma(\vec{k}, j)=-\frac{\partial[\ln \omega(\vec{k}, j)]}{\partial \ln V}=-\frac{V}{\omega(\vec{k}, j)} \frac{\partial \omega(\vec{k}, j)}{\partial V} .
$$

The partial derivative is evaluated at constant temperature and number of particles. Within the quasiharmonic approximation the Helmholtz free energy of a crystal can be expressed as ${ }^{37}$

$$
F(V, T)=E(V)+F_{p h}(V, T),
$$

where $E(V)$ is the energy of the motionless lattice obtained directly from $a b$ initio calculations while $F_{p h}(V, T)$ denotes the vibrational free energy of a harmonic system. The term $F_{p h}(V, T)$ includes the vibrational zero-point energy which remains finite for $T \rightarrow 0$. Any purely electronic contribution is neglected. One has to note that only $F_{p h}(V, T)$ depends explicitly on temperature. At a given temperature $T$, the equilibrium volume follows from a minimization of $F(V, T)$ with respect to $V$. This enables to determine the volume thermalexpansion coefficient $\alpha_{V}(T)=\frac{1}{V}\left(\frac{\partial V}{\partial T}\right)_{p}$ (see Ref. 37). An as- 
TABLE I. Calculated and experimental (Ref. 40) elastic constants $\left(C_{i j}\right)$ for $\mathrm{ReO}_{3}$ crystal. Data are given in gigapascal. The numbers quoted in brackets denote experimental errors. The bulk modulus can be obtained as $B=\left(C_{11}+2 C_{12}\right) / 3$.

\begin{tabular}{lcc}
\hline \hline$C_{i j}$ & Theory & Exp. (78 K) \\
\hline$C_{11}$ & 608 & $609(3)$ \\
$C_{12}$ & 74 & $12(14)$ \\
$C_{44}$ & 66 & $69(0)$ \\
\hline \hline
\end{tabular}

sumption that the number of particles is kept constant together with pressure applies. In the quasiharmonic approximation $\alpha_{V}(T)$ can be obtained from the following Grüneisen relation: ${ }^{37}$

$$
\gamma(T)=\frac{V \alpha_{V} B_{T}}{C_{V}},
$$

where $B_{T}$ is the isothermal bulk modulus while $C_{V}$ stands for the lattice contribution to the total heat capacity at the constant volume. Symbol $\gamma(T)$ denotes the overall Grüneisen parameter being the weighted average of the mode-specific Grüneisen parameters, i.e., $\quad \gamma(T)$ $=\sum_{\vec{k}, j} \gamma(\vec{k}, j) C_{V}(\vec{k}, j) / \sum_{\vec{k}, j} C_{V}(\vec{k}, j)$ with $C_{V}(\vec{k}, j)$ describing the $(\vec{k}, j)$ mode contribution to $C_{V}$ (see Ref. 37). Technical details concerning implementations of the quasiharmonic approximation in ab initio codes as well as determination of the mode-specific Grüneisen parameters can be found, for example, in Ref. 38.

In the present work, a comparison between experimentally determined and calculated GDOS is made. For a given temperature $T$, the GDOS is evaluated from Eq. (4) for a numerical $\mathrm{ReO}_{3}$ sample using the structure calculated at the equilibrium $V(T)$ given by the quasiharmonic approximation. Experimental and calculated GDOS are normalized to unity, i.e., $\int_{0}^{\infty} d \omega G(\omega)=1$.

\section{RESULTS AND DISCUSSION}

The ground-state lattice constant of $\mathrm{ReO}_{3}$ crystal obtained within the LDA approximation amounts to $3.7467 \AA$ (Ref. 39). It remains in a very good agreement with the experimental value of $3.7477 \AA$ measured at low temperatures. ${ }^{15}$ The calculated bulk modulus of $\mathrm{ReO}_{3}$ determined from the stressstrain relations equals $252 \mathrm{GPa}$ as compared to the experimental value of $211 \mathrm{GPa}$ obtained at low temperatures by Tsuda et al. ${ }^{40}$ Theoretical and experimental elastic constants are listed in Table I. The calculated component $C_{12}$ is highly overestimated in comparison with the experimental value. On the other hand, one has to note that the experimental $C_{12}$ is determined with a quite large error. It should be also mentioned that the LDA approximation is well known to produce overestimated bulk modulus. Substantial difference found between $C_{11}$ and $C_{12}$ in the $\mathrm{ReO}_{3}$ crystal results in a high anisotropy of this compound being characterized by the anisotropy factor of 0.23 .

The primitive unit cell of $\mathrm{ReO}_{3}$ crystal contains four atoms which give rise to 12 phonon branches. At the Brillouin

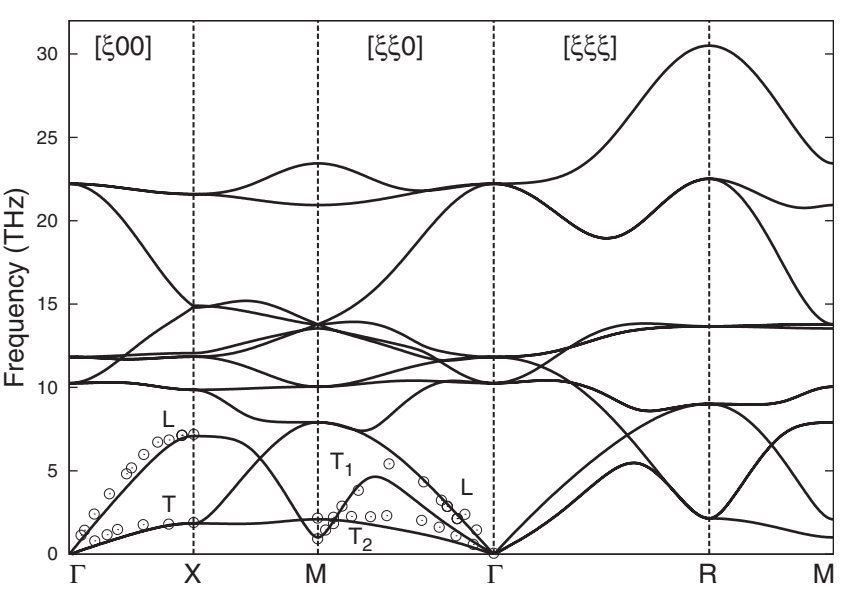

FIG. 3. Ab initio calculated phonon-dispersion curves in $\mathrm{ReO}_{3}$ at ambient pressure. Experimental data from inelastic neutron scattering (Ref. 21) measured at room temperature are shown as open symbols.

zone center the threefold degenerate modes $T_{1 u}$ and $T_{2 u}$ are observed. The mode $T_{1 u}$ consists of $T_{1 u}^{(1)}, T_{1 u}^{(2)}$, and $T_{1 u}^{(3)}$ components which are infrared active while the mode $T_{2 u}$ is silent. The phonon-dispersion relations along high-symmetry points calculated at ambient pressure are shown in Fig. 3. They are compared to the experimental data measured at room temperature by inelastic neutron scattering. ${ }^{21}$ It should be noted that experimental branches were obtained only for the acoustic region and along the $[\xi 00]$ and $[\xi \xi 0]$ directions. Both the longitudinal acoustic and transverse acoustic (TA) modes are well reproduced by the present calculations. The TA branches along $[\xi 00]$ and $[\xi \xi 0]$ directions are rather flat. They have very low frequencies extending up to the zone boundaries. Such a feature is typical for the perovskite-type oxides. $^{41,42}$ The $T_{1}(\xi \xi 0)$ mode is much more dispersive than $T_{2}(\xi \xi 0)$ mode and it undergoes a pronounced softening near the $M$ point. One can also notice a softening of TA branch along $[\xi \xi \xi]$ direction while approaching the $R$ point. Calculated frequencies of modes at $\Gamma, M$, and $R$ points of the Brillouin zone calculated at ambient pressure are collected in Table II. Figure 4 shows the calculated phonon DOS at ambient pressure and zero temperature. Partial phonon densities of states from $\mathrm{Re}$ and $\mathrm{O}$ atoms contributing to the total DOS

TABLE II. Calculated and experimental frequencies of phonons at $\Gamma, M$, and $R$ points in $\mathrm{ReO}_{3}$ crystal at ambient pressure. Frequencies are given in terahertz.

\begin{tabular}{lccc}
\hline \hline Mode & Theory & \multicolumn{2}{c}{ Experiments } \\
\hline & & Ref. 28 & Ref. 21 \\
& & $150 \mathrm{~K}$ & $300 \mathrm{~K}$ \\
$M \equiv T_{1}\left(\frac{1}{2}, \frac{1}{2}, 0\right)$ & 1.007 & 0.822 & 0.933 \\
$R \equiv T\left(\frac{1}{2}, \frac{1}{2}, \frac{1}{2}\right)$ & 2.146 & \\
\multicolumn{4}{c}{$\Gamma$ point } \\
$T_{1 u}^{(1)}$ & 10.255 & \\
$T_{1 u}^{(2)}$ & 22.217 & \\
$T_{2 u}$ & 11.825 & \\
\hline \hline
\end{tabular}




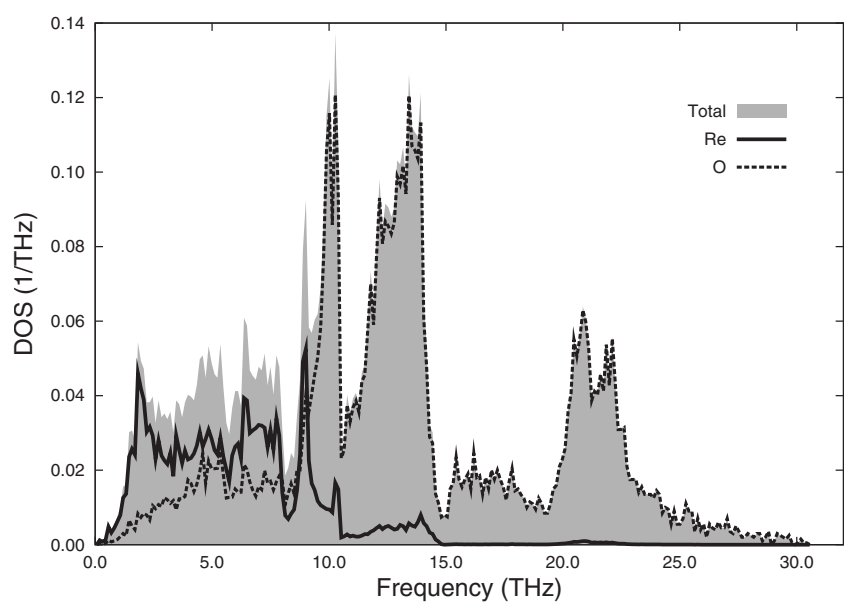

FIG. 4. Calculated phonon density of states of $\mathrm{ReO}_{3}$ at ambient pressure. Contributions to total DOS (shaded area) from Re and $\mathrm{O}$ atoms are denoted by solid and dashed lines, respectively.

are shown as well. They indicate that in $\mathrm{ReO}_{3}$ crystal the high-frequency optical phonons are dominated by the vibrations of oxygen atoms. In this range of frequencies Re atoms contribute very little to the lattice vibrations. The low-lying phonons are mainly due to the mixed vibrations of $\mathrm{Re}$ and $\mathrm{O}$ atoms. Here, a quite significant contribution comes also from oxygen vibrations.

In many materials exposed to an external pressure one usually observes an increase in phonon frequencies upon crystal compression. This effect is mainly due to a decrease in the crystal volume with the increasing pressure which involves a diminishing of the interatomic distances and an increase in the force constants. In $\mathrm{ReO}_{3}$ crystal, such a behavior is encountered for the zone-center optical phonon mode frequencies. These frequencies shift slightly upward with the increasing pressure, as shown in the inset of Fig. 5. Here we show only the modes having nonzero frequencies. No comparison is made neither to experiment nor other ab initio calculations due to the lack of such data for optical phonons in $\mathrm{ReO}_{3}$ compound. Below 4 kbar the $T_{1 u}^{(2)}$ and $T_{1 u}^{(3)}$ modes increase almost linearly with pressure while the $T_{2 u}$ modes, after an initial growth, remain unchanged upon compression. Generally, at pressures below 4 kbar the relative changes in the mode frequencies of $\mathrm{ReO}_{3}$ are very small and they do not exceed $0.5 \%$. The effect of pressure on a given phonon mode can be described in terms of the mode Grüneisen parameter, defined by Eq. (6). The calculated Grüneisen parameters for $T_{1 u}^{(2)}, T_{1 u}^{(3)}$, and $T_{2 u}$ modes are positive and they amount to $1.24,2.11$, and 1.03 , respectively.

In most of the perovskite-type compounds, the $M$ and $R$ phonon modes which are associated, respectively, with inphase and out-of-phase rigid rotations of the octahedra about [100]-type axes are unstable versus pressure. These rotational modes gradually condense under crystal compression. Condensation of these modes was established to be the main driving force of the different phase transformations encountered in perovskites. ${ }^{43,44}$ Present calculations show that the $M$ and $R$ phonons in $\mathrm{ReO}_{3}$ soften simultaneously upon increasing pressure (see Fig. 5). The $M$ mode softens more rapidly than the $R$ mode and the $M$ mode is expected to

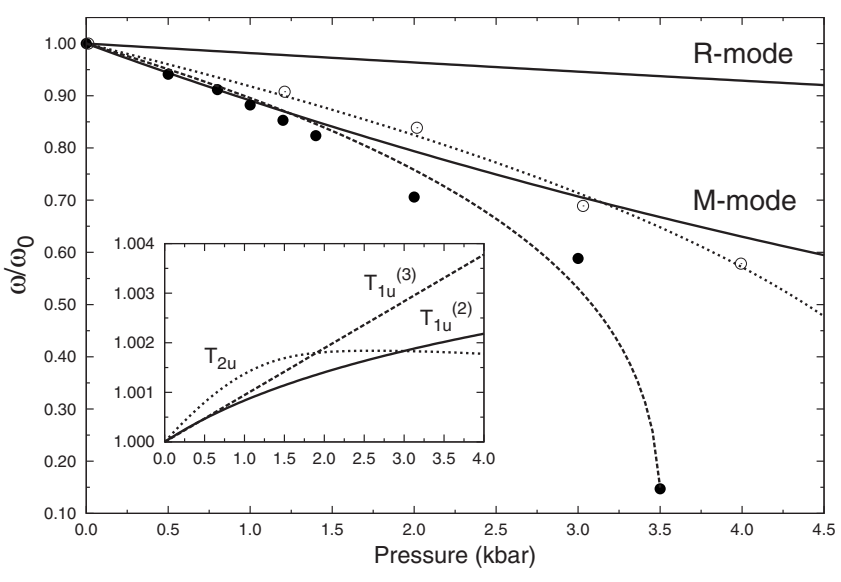

FIG. 5. Pressure dependence of the $M\left(\frac{1}{2}, \frac{1}{2}, 0\right)$-point and $R\left(\frac{1}{2}, \frac{1}{2}, \frac{1}{2}\right)$-point phonon frequencies in $\mathrm{ReO}_{3}$ crystal. Experimental data for $M$ mode are taken from Ref. 28 (solid symbols) and Ref. 21 (open symbols). Solid lines represent $a b$ initio calculations. Dashed and dotted curves denote approximations of the experimental data. Inset: calculated frequencies of optical modes at the $\Gamma$ point. Modes $T_{1 u}^{(1)}, T_{1 u}^{(2)}$, and $T_{2 u}$ are indicated by solid, dashed, and dotted curves, respectively. Ambient pressure frequencies of particular modes $\left(\omega_{0}\right)$ are given in Table II.

condense. The softening of these modes can be to a good approximation described by the exponential decay law with the softening rates of -0.12 and $-0.04 \mathrm{THz} / \mathrm{kbar}$ for the $M$ and $R$ modes, respectively. These phonon modes which soften with crystal compression are characterized by the negative Grüneisen constants. The calculated Grüneisen parameters of the $M$ and $R$ modes amount to -291 and -46 , respectively. For pressures not exceeding 1 kbar, experimental data of Chatterji et al. ${ }^{28}$ and Axe et al..$^{21}$ yield the $M$ mode Grüneisen constants of -242 and -180 , respectively. The large negative values of the Grüneisen constants which are found either for the $M$ or $R$ phonon mode lead to the negative thermal expansion at low temperatures where these modes dominate.

According to the quasiharmonic approximation, the thermal expansion of the $\mathrm{ReO}_{3}$ crystal can be directly determined from the calculated free energy given by Eq. (7). The minimum of the $F(V, T)$ curve determines the equilibrium volume at a given $T$. The relative change in the $\mathrm{ReO}_{3}$ volume with temperature is shown in Fig. 6. A comparison is made to the experimental data reported in Refs. 15 and 16. Although a decrease in the $\mathrm{ReO}_{3}$ volume with temperature is predicted by the quasiharmonic approximation, an agreement between theory and experiment is only qualitative. Our calculations produce rather shallow minimum as compared to that obtained from the measurements. Moreover, calculations yield the negative thermal expansion up to $170 \mathrm{~K}$ while the neutron-scattering experiments reveal NTE behavior up to $200 \mathrm{~K}$. At higher temperatures, both theory and experiment show a gradual increase in the $\mathrm{ReO}_{3}$ volume. The lattice dynamical calculations ${ }^{15}$ based on the shell model find a minimum of the $\mathrm{ReO}_{3}$ volume at about $350 \mathrm{~K}$ and the positive thermal expansion extending up to high temperatures. The calculated volumetric thermal-expansion coefficient, shown in the inset of Fig. 6, varies between -2.1 


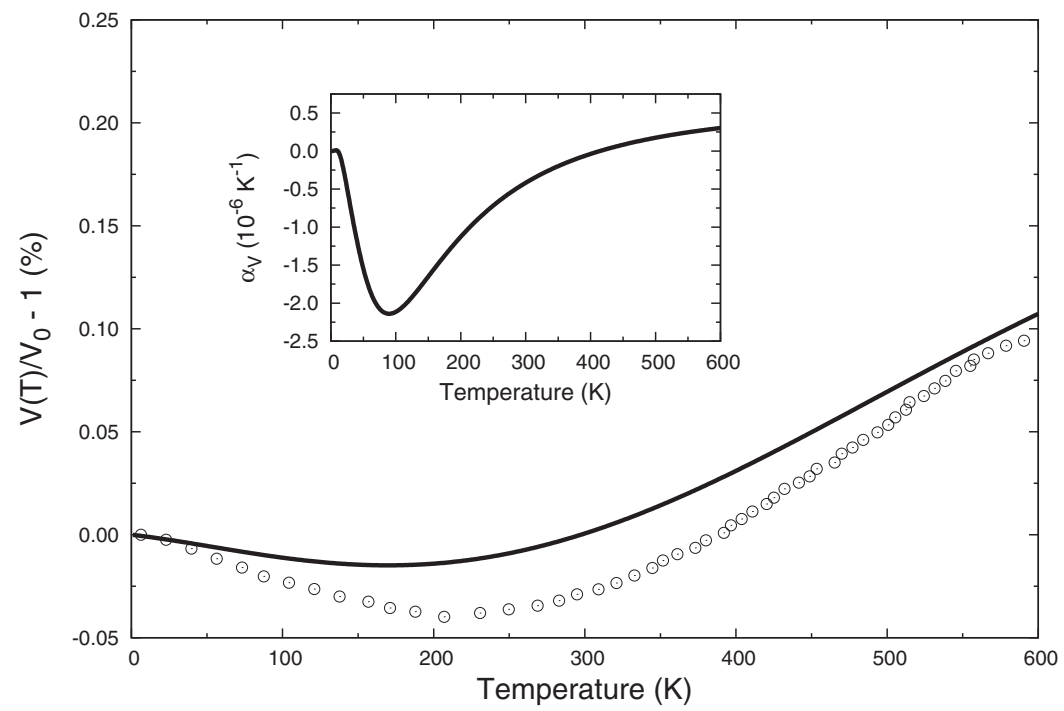

FIG. 6. Calculated and experimental evolution of the $\mathrm{ReO}_{3}$ volume with temperature. Solid line represents the quasiharmonic approximation calculations. Open symbols indicate experimental data taken from Refs. 15 and 16. Inset: temperature dependence of the calculated volumetric thermal-expansion coefficient using the Grüneisen relationship [Eq. (8)].

$\times 10^{-6} \mathrm{~K}^{-1}$ and zero in the NTE regime and the average value of the thermal expansivity below the NTE to PTE crossover temperature equals $-1.2 \times 10^{-6} \mathrm{~K}^{-1}$. At high temperatures the quasiharmonic theory gives about two times lower values of $\alpha_{V}$ as compared to the shell model calculations. On the other hand, both approaches yield very similar values of the thermal expansivity below the respective NTE to PTE crossover temperature.

Neutron-scattering experiments carried out either by Chatterji et al. ${ }^{15}$ or very recently by Rodriguez et al. ${ }^{31}$ point out that a static disorder due to oxygen atoms can play a significant role in the negative thermal expansion of $\mathrm{ReO}_{3}$ compound. The samples with a large contribution from a static disorder were found to exhibit diminished NTE while those with less static disorder showed a high NTE to PTE crossover temperature $(294 \mathrm{~K})$ and a quite large negative thermal expansivity $\left(-1.0 \times 10^{-6} \mathrm{~K}^{-1}\right)$. Additional information about the mechanisms responsible for the NTE phenomenon in $\mathrm{ReO}_{3}$ can be gained from the mean-squared vibrational amplitudes of $\mathrm{Re}$ and $\mathrm{O}$ atoms. The mean-squared displacements $\left(U_{i j}\right)$ of a given atom constitute a second-rank symmetric tensor and they can be expressed by the diagonal and off-diagonal partial phonon densities of states. ${ }^{36,45}$ They enter the Debye-Waller factor describing an intensity of the radiation scattered by a crystal. Experimental $U_{i j}$ contain contributions from both static and dynamic displacements of atoms. Here, we limit our consideration to a dynamic component and the harmonic approximation. The site symmetry of Re atom $(\overline{3} m)$ constrains its mean-squared displacements to be isotropic and hence described by a single parameter. Oxygen atoms in $\mathrm{ReO}_{3}$ posses site symmetry of $4 / \mathrm{mmm}$ leading to anisotropic thermal motion. At the oxygen site, the $U_{i j}$ tensor has two independent components, namely, $U_{11}$ and $U_{22}=U_{33}$ which represent oxygen vibrations parallel and perpendicular to the Re-O bond, respectively. A comparison of the calculated and measured ${ }^{15}$ mean-squared displacements is shown in Fig. 7. It seems obvious that Re atoms exhibit smaller amplitude of their thermal motion than $\mathrm{O}$ atoms and one could expect that a difference in the respective meansquared displacements should be mainly due to the difference between the mass of rhenium and that of oxygen. This is, however, not confirmed neither by theoretical nor experimental results which show that $U_{11}(\mathrm{O})$ and $U(\mathrm{Re})$ are of the same order of magnitude and become comparable at elevated temperatures. This effect is likely to be driven by a difference in the respective force constants. Indeed, our calculations show that the force constant at the Re site is only 1.3 times higher than the force constant, $\Phi_{x x}(\mathrm{O})$, associated with the $U_{11}(\mathrm{O})$ component. This suppresses the effect connected with a large difference between the mass of the cation and anion in $\mathrm{ReO}_{3}$ and leads to a diminished difference between respective thermal motions when the temperature is raised. Despite a deviation seen between the calculated and measured $U_{11}(\mathrm{O})$, which is enhanced with increasing temperature, a qualitative agreement between the harmonic approximation and the experiment is achieved. This, however, does not apply to $U_{33}(\mathrm{O})$ component, which remains highly underestimated as compared to that determined in experiments. It should be mentioned that the experimental $U_{33}(\mathrm{O})$ is not reproduced by the lattice dynamical calculations ${ }^{15}$ either.

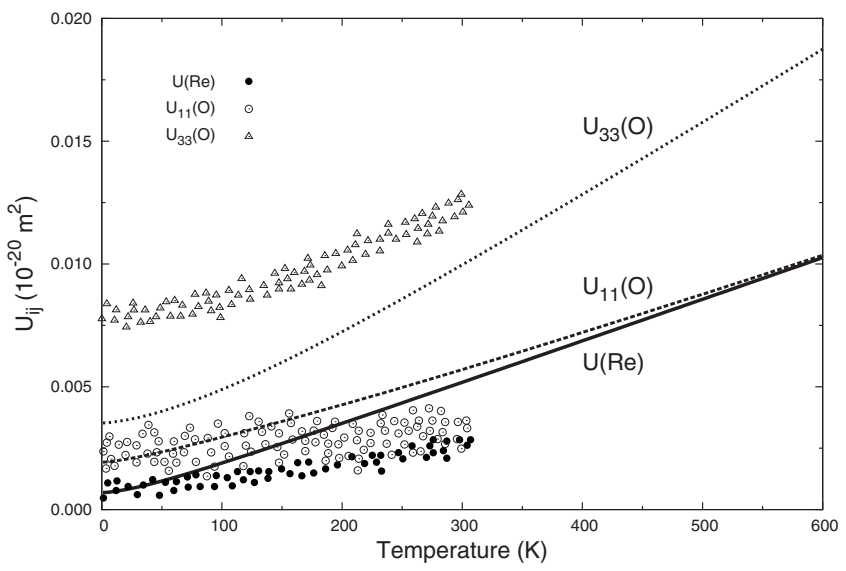

FIG. 7. Calculated and experimental mean-squared displacement versus temperature for $\mathrm{Re}$ and $\mathrm{O}$ atoms in $\mathrm{ReO}_{3}$ crystal. Calculated $U_{11}$ and $U_{33}$ components for oxygen atoms are shown by dashed and dotted curves, respectively. Isotropic thermal displacements of rhenium atoms are indicated by solid curve. Experimental data for Re (solid symbols) and $\mathrm{O}$ (open symbols) are adopted from Ref. 15. 
Both theoretical approaches, remaining in quantitative agreement with each other, indicate for $U_{33}(\mathrm{O})$ to be strongly temperature dependent. The oxygen vibrations perpendicular to $\mathrm{Re}-\mathrm{O}$ bond are characterized by nearly two times larger slope than oxygen vibrations along the bond.

Some explanation of the observed disagreement between calculated and measured $U_{33}(\mathrm{O})$ has been already proposed. ${ }^{15}$ This deviation was assigned to some kind of static disorder experienced by those oxygen atoms which are perpendicular to the $\mathrm{Re}-\mathrm{O}$ bond. This assumption was subsequently, and at least partly, supported by the neutronscattering experiments carried out on powder samples having various qualities. ${ }^{31}$ The higher quality samples (prepared by crushing single crystals of $\mathrm{ReO}_{3}$ ) were found to reveal a diminished anisotropy, $\eta=U_{33}(\mathrm{O}) / U_{11}(\mathrm{O})$, in oxygen thermal vibrations perpendicular and parallel to the $\mathrm{Re}-\mathrm{O}$ bond, i.e., a lower gap between $U_{33}(\mathrm{O})$ and $U_{11}(\mathrm{O})$. Although the absolute magnitudes of the calculated thermal displacements still remain underestimated as compared with those measured for the best-quality sample, nevertheless at low temperatures theoretical $\eta$ retains the value obtained in experiment. ${ }^{31}$ In addition, the measured mean-squared vibrations are shifted upward with respect to the calculated vibrations, i.e., the observed mismatch is predominantly due to a static component which is not taken into account in our $a b$ initio calculations as they concern perfectly ordered and undistorted $\mathrm{ReO}_{3}$ crystal.

Our calculations show that the anisotropy in the oxygen thermal motion can be related with the anisotropy of the oxygen force constants in the Re-O bond direction and perpendicular to it. Indeed, the calculated force constant $\Phi_{z z}(\mathrm{O})$ which is associated with the $U_{33}(\mathrm{O})$ component is found to be over twice weaker than $\Phi_{x x}(\mathrm{O})$. This allows for a larger amplitude of the thermal vibrations in the direction perpendicular to the $\mathrm{Re}-\mathrm{O}$ bond than in the direction along the bond. It also should be pointed out that the thermal motion of oxygen atoms is highly anisotropic in a direction which is consistent with the atomic displacements giving rise to the $M$ phonon mode, the latter driven the coordinated rotations of the $\mathrm{ReO}_{3}$ octahedra around the [100]-type axes.

Figure 8 shows the temperature dependence of the anisotropy in the thermal motion of oxygen atoms calculated for $\mathrm{ReO}_{3}$ crystal. It can be noticed that the thermal vibrations of oxygen atoms are anisotropic at every temperature. This anisotropy decreases initially with increasing temperature and reaches a minimum at about $100 \mathrm{~K}$. One has to note that both $\eta$ and $\alpha_{V}$ have very similar courses versus temperature with the thermal expansivity showing also a minimum in the vicinity of $100 \mathrm{~K}$ (Fig. 6). Therefore, it seems that the NTE phenomenon in $\mathrm{ReO}_{3}$ coincide with the more isotropic thermal motion of oxygen atoms. Thus, a reduction or enhancement in $\eta$ can be an additional driving force which tends to convert $\mathrm{ReO}_{3}$ system from NTE to PTE and vice verse. The inset of Fig. 8 indicates that $\eta$ slightly increases with $\mathrm{ReO}_{3}$ compression and such a behavior is encountered at every temperature. This effect results from a simultaneous hardening of $\Phi_{x x}(\mathrm{O})$ and softening of $\Phi_{z z}(\mathrm{O})$ with the applied pressure which lead, respectively, to reduction in $U_{11}(\mathrm{O})$ and enhancement of $U_{33}(\mathrm{O})$, and finally to the observed increase in $\eta$ with crystal compression.

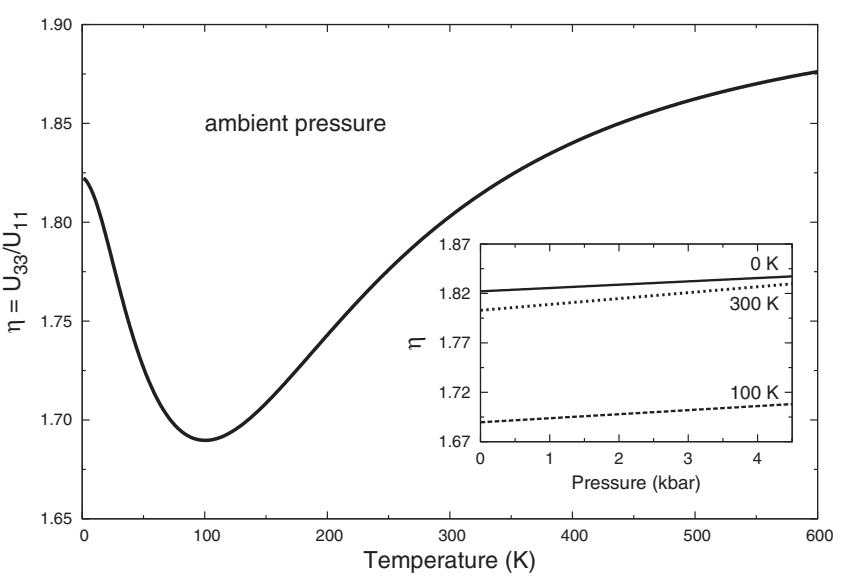

FIG. 8. Calculated temperature dependence of the anisotropy $(\eta)$ in oxygen thermal motion for $\mathrm{ReO}_{3}$ crystal. Inset: pressure dependence of anisotropy at temperatures $T=0 \mathrm{~K}$ (solid line), $T$ $=100 \mathrm{~K}$ (dashed line), and $T=300 \mathrm{~K}$ (dotted line).

The slope of the theoretical mean-squared displacements could be used to estimate the Debye temperature $\left(\Theta_{D}\right)$ of the $\mathrm{ReO}_{3}$ crystal. The calculated $\Theta_{D}$ amounts to $353 \mathrm{~K}$. This value remains in a good agreement with $\Theta_{D}=327 \mathrm{~K}$ determined from the specific-heat capacity experiments ${ }^{10}$ and electrical-conductivity measurements. ${ }^{11}$

A comparison between experimental and calculated generalized form of the phonon densities of states is shown in Fig. 9. The calculated contributions to GDOS from Re and O atoms are shown as well. It should be noted that $\mathrm{Re}$ and $\mathrm{O}$ atoms have significantly different scattering efficiencies $\left(\alpha_{\mathrm{Re}}=0.062 \mathrm{barn} / \mathrm{amu}\right.$ and $\left.\alpha_{\mathrm{O}}=0.265 \mathrm{barn} / \mathrm{amu}\right)$. Therefore, the resulted neutron-weighted partial phonon densities of states are different than the bare partial densities of states presented in Fig. 4. In this case, the scattering is dominated by the oxygen contribution (93\%) and the experimental GDOS can be directly compared to the oxygen partial density of states. The GDOS looks as if the contributions of oxygen partial density of states were too strong. Indeed,

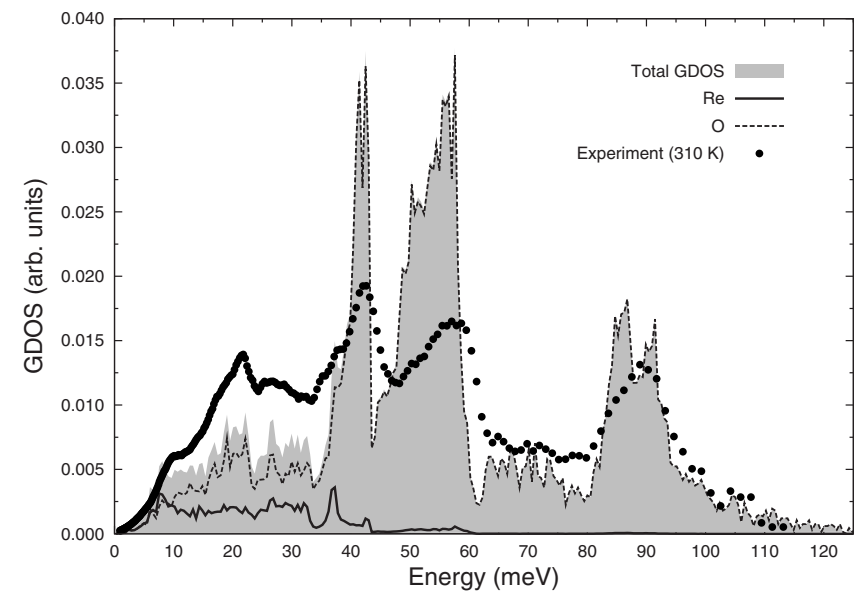

FIG. 9. Comparison between measured (solid symbols) and calculated (shaded area) GDOSs for $\mathrm{ReO}_{3}$ sample at $310 \mathrm{~K}$. Contributions from $\mathrm{Re}$ and $\mathrm{O}$ vibrations to GDOS are shown by solid and dashed curves, respectively. 
there are two high peaks at 41 and $55 \mathrm{meV}$, which seem to be less intense in the measured GDOS. Moreover, the lowenergy part of the calculated intensity in the interval from 10 till $35 \mathrm{meV}$ remains underestimated. This behavior could be explained by taking into account the suggested static disorder of oxygen atoms which is revealed in their thermal displacements. A static disorder may break the propagation of phonons over the crystal lattice and give rise to changes in the density of states in such a way that the relatively high intensity due to the mentioned peaks at 41 and $55 \mathrm{meV}$ is shifted into the rather continuous distribution at the lowenergy interval of the GDOS.

\section{SUMMARY AND CONCLUSIONS}

The structural, vibrational, and thermodynamic properties of $\mathrm{ReO}_{3}$ crystal has been studied by the quasiharmonic approximation with the density-functional theory. Dispersion relations of phonons at ambient pressure show good agreement with the inelastic neutron-scattering experiments. The zone-center phonon mode frequencies increase slightly with the increasing pressure and they are characterized by the positive Grüneisen parameters. Large negative values of the Grüneisen constants are calculated for the acoustic modes at the $M$ and $R$ high-symmetry points of the Brillouin zone of $\mathrm{ReO}_{3}$. During the crystal compression the $M$ phonon softens more rapidly than the $R$ phonon. For pressures lower than 2 kbar the softening rate of the $M$ phonon remains in a reasonable agreement with experiments.
Oxygen atoms in the $\mathrm{ReO}_{3}$ crystal have highly anisotropic thermal vibrations which follow from the anisotropy of the force constants at the oxygen site. This anisotropy is also consistent with the $M$ phonon and it contributes to the negative thermal expansivity. When the oxygen atoms perform the more isotropic thermal motion, the NTE phenomenon becomes enhanced. The thermal anisotropy at the oxygen site can be an additional force driving the low-pressure phase transition in $\mathrm{ReO}_{3}$ since it increases upon crystal compression. The calculated and experimental room-temperature generalized phonon densities of states remain in a very good agreement.

The quasiharmonic theory seems to be insufficient to give the experimental magnitudes of some dynamical and thermal quantities for the $\mathrm{ReO}_{3}$ crystal. A discrepancy remains pronounced especially for these crystal properties which are more strongly affected by intrinsic crystal defects, such as static disorder. In principle, the latter issue could be to some extent taken into account by ab initio methods, however, such simulations would require some model structure to be assumed. On the other hand, one has to remember that calculations of lattice dynamics for compounds with nonperfect structural environment ${ }^{45}$ are highly demanding especially for first-principles methods using a supercell approach.

\section{ACKNOWLEDGMENTS}

Interdisciplinary Modeling Center ICM, Warsaw University, Poland is acknowledged for providing the computer facilities to support part of the present calculations under Grant No. G28-12.
${ }^{1}$ W. Miller, C. W. Smith, D. S. Mackenzie, and K. E. Evans, J. Mater. Sci. 44, 5441 (2009).

${ }^{2}$ Y. M. Hao, Y. Gao, B. W. Wang, J. P. Qu, Y. X. Li, F. Hu, and J. C. Deng, Appl. Phys. Lett. 78, 3277 (2001).

${ }^{3}$ J. R. Salvador, F. Gu, T. Hogan, and M. G. Kanatzidis, Nature (London) 425, 702 (2003).

${ }^{4}$ J. Arvanitidis, K. Papagelis, S. Margadonna, K. Prassides, and A. N. Fitch, Nature (London) 425, 599 (2003).

${ }^{5}$ T. A. Mary, J. S. O. Evans, T. Vogt, and A. W. Sleight, Science 272, 90 (1996).

${ }^{6}$ A. Sanson, F. Rocca, G. Dalba, P. Fornasini, R. Grisenti, M. Dapiaggi, and G. Artioli, Phys. Rev. B 73, 214305 (2006).

${ }^{7}$ R. Mittal, S. L. Chaplot, H. Schober, and T. A. Mary, Phys. Rev. Lett. 86, 4692 (2001).

${ }^{8}$ R. Mittal, S. L. Chaplot, H. Schober, A. I. Kolesnikov, C.-K. Loong, C. Lind, and A. P. Wilkinson, Phys. Rev. B 70, 214303 (2004).

${ }^{9}$ J. W. Zwanziger, Phys. Rev. B 76, 052102 (2007).

${ }^{10}$ C. N. King, H. C. Kirsch, and T. H. Geballe, Solid State Commun. 9, 907 (1971).

${ }^{11}$ T. Tanaka, T. Akahane, E. Bannai, S. Kawai, N. Tsuda, and Y. Ishizawa, J. Phys. C 9, 1235 (1976).

${ }^{12}$ L. F. Mattheiss, Phys. Rev. 181, 987 (1969).

${ }^{13}$ M. Morinaga, K. Sato, J. Harada, H. Adachi, S. Ohba, and Y. Saito, J. Phys. C 16, L177 (1983).
${ }^{14}$ F. Corà, M. G. Stachiotti, and C. R. A. Catlow, J. Phys. Chem. B 101, 3945 (1997); M. G. Stachiotti, F. Corà, C. R. A. Catlow, and C. O. Rodriguez, Phys. Rev. B 55, 7508 (1997).

${ }^{15}$ T. Chatterji, P. F. Henry, R. Mittal, and S. L. Chaplot, Phys. Rev. B 78, 134105 (2008).

${ }^{16}$ T. Chatterji, T. C. Hansen, M. Brunelli, and P. F. Henry, Appl. Phys. Lett. 94, 241902 (2009).

${ }^{17}$ F. S. Razavi, Z. Altounian, and W. R. Datars, Solid State Commun. 28, 217 (1978).

${ }^{18}$ J. E. Schirber and B. Morosin, Phys. Rev. Lett. 42, 1485 (1979).

${ }^{19}$ J. E. Schirber and L. F. Mattheiss, Phys. Rev. B 24, 692 (1981).

${ }^{20}$ B. Batlogg, R. G. Maines, M. Greenblatt, and S. DiGregorio, Phys. Rev. B 29, 3762 (1984).

${ }^{21}$ J. D. Axe, Y. Fujii, B. Batlogg, M. Greenblatt, and S. DiGregorio, Phys. Rev. B 31, 663 (1985).

${ }^{22}$ J.-E. Jørgensen, J. D. Jorgensen, B. Batlogg, J. P. Remeika, and J. D. Axe, Phys. Rev. B 33, 4793 (1986).

${ }^{23}$ J. E. Schirber, B. Morosin, R. W. Alkire, A. C. Larson, and P. J. Vergamini, Phys. Rev. B 29, 4150 (1984).

${ }^{24}$ J. E. Jørgensen, S. Olsen, and L. Gerward, J. Appl. Crystallogr. 33, 279 (2000).

${ }^{25}$ E. Suzuki, Y. Kobayashi, S. Endo, and T. K. Ikegawa, J. Phys.: Condens. Matter 14, 10589 (2002).

${ }^{26}$ J. E. Jørgensen, W. G. Marshall, R. I. Smith, J. Staun Olsen, and L. Gerward, J. Appl. Crystallogr. 37, 857 (2004). 
${ }^{27}$ T. Chatterji and G. J. McIntyre, Solid State Commun. 139, 12 (2006).

${ }^{28}$ T. Chatterji, P. G. Freeman, M. Jimenez-Ruiz, R. Mittal, and S. L. Chaplot, Phys. Rev. B 79, 184302 (2009).

${ }^{29}$ N. Matsuno, M. Yoshimi, S. Ohtake, T. Akahane, and N. Tsuda, J. Phys. Soc. Jpn. 45, 1542 (1978).

${ }^{30}$ J. Purans, G. Dalba, P. Fornasini, A. Kuzmin, S. De Panfilis, and F. Rocca, XAFS13: International Conference on X-ray Absorption Fine Structure (AIP, New York, 2007), Vol. 882, pp. 422424.

${ }^{31}$ E. E. Rodriguez, A. Llobet, T. Proffen, B. C. Melot, R. Seshadri, P. B. Littlewood, and A. K. Cheetham, J. Appl. Phys. 105, 114901 (2009).

${ }^{32}$ For example, see S. W. Lovesey, Theory of Neutron Scattering from Condensed Matter (Oxford University Press, New York, 1987), Chap. 4.

${ }^{33}$ W. Reichardt, MUPHOCOR, a Fortran Program to determine the Phonon Density of States from Neutron Scattering Experiments (Karlsruhe Institute for Nuclear Physics, Karlsruhe, 1984).

${ }^{34}$ V. S. Oskotskii, Sov. Phys. Solid State 9, 420 (1967).

${ }^{35} \mathrm{G}$. Kresse and J. Furthmüller, computer code VASP, Vienna, Austria, 1999; Phys. Rev. B 54, 11169 (1996).
${ }^{36}$ K. Parlinski, software PHONON, Cracow, Poland, 2008; K. Parlinski, Z.-Q. Li, and Y. Kawazoe, Phys. Rev. Lett. 78, 4063 (1997).

${ }^{37}$ D. C. Wallace, Thermodynamics of Crystals (Wiley, New York, 1972); N. W. Ashcroft and N. D. Mermin, Solid State Physics (Holt, Rinehart and Winston, New York, 1976).

${ }^{38}$ G. J. Ackland, X. Huang, and K. M. Rabe, Phys. Rev. B 68, 214104 (2003); A. Siegel, K. Parlinski, and U. D. Wdowik, ibid. 74, 104116 (2006); G. Kern, G. Kresse, and J. Hafner, ibid. 59, 8551 (1999).

${ }^{39}$ Test calculations performed with another PAW pseudopotential for Re atom, $\left(5 p^{6} 6 s^{2} 5 d^{5}\right)$ configuration, taken from the VASP database lead to a very similar value of the lattice constant (3.7474 ̊).

${ }^{40}$ N. Tsuda, Y. Sumino, I. Ohno, and T. Akahane, J. Phys. Soc. Jpn. 41, 1153 (1976).

${ }^{41}$ J. D. Axe, J. Harada, and G. Shirane, Phys. Rev. B 1, 1227 (1970).

${ }^{42}$ M. Sato, B. Grier, G. Shirane, and T. Akahane, Phys. Rev. B 25, 6876 (1982).

${ }^{43}$ K. Parlinski and Y. Kawazoe, Eur. Phys. J. B 16, 49 (2000).

${ }^{44}$ W. Yu, J. Zhao, and C. Jin, Phys. Rev. B 72, 214116 (2005).

${ }^{45}$ U. D. Wdowik and K. Parlinski, Phys. Rev. B 78, 224114 (2008); J. Phys.: Condens. Matter 21, 125601 (2009). 